\title{
ARTICLE Nicotinamide adenine dinucleotide replenishment rescues colon degeneration in aged mice
}

\author{
Xudong Zhu ${ }^{1}$, Weiyan Shen ${ }^{1}$, Ying Wang ${ }^{1}$, Amit Jaiswal ${ }^{1,2}$, Zhenyu Ju ${ }^{1}$ and Qinsong Sheng ${ }^{3}$
}

Susceptibility of gastrointestinal dysmotility increases with age-associated colonic degeneration. A paucity of remedies reversing colonic degeneration per se hinders the fundamental relief of symptoms. Here we discovered the correlation between colon degeneration and altered nicotinamide adenine dinucleotide (NAD) level in aged mice. Compared to 3-month-old young controls, 2-year-old mice showed a spectrum of degenerative colonic phenotypes and exhibited a significant elongated transit time and slowed stool frequency in the context of Lomotil-induced slow-transit constipation. Despite upregulated colonic tryptophan hydroxylases expression, serotonin release and expression of colon-predominant type IV serotonin receptor, reduced viability of interstitial cells of Cajal while enhanced aquaporins (Aqp1, 3 and 11) led to a less colonic motility and increased luminal dehydration in aged mice. Notably, this colonic degeneration was accompanied with reduced key NAD ${ }^{+}$-generating enzyme expression and lowered $\mathrm{NAD}^{+} / \mathrm{NADH}$ ratio in aged colon. Three-month continuous administration of beta nicotinamide mononucleotide, a NAD ${ }^{+}$precursor, elevated colonic $\mathrm{NAD}^{+}$level and improved defecation in aged mice. In contrast, pharmacological inhibition of nicotinamide phosphoribosyltransferase, the rate-limiting enzyme for $\mathrm{NAD}^{+}$biosynthesis, induced a reduction in colonic NAD content and impaired gastrointestinal function in young mice. Taken together, these findings suggest the beneficial effect of $\mathrm{NAD}^{+}$in maintaining colonic homoeostasis and reactivating $\mathrm{NAD}^{+}$biosynthesis may represent a promising strategy to counteract age-related gastrointestinal degeneration.

Signal Transduction and Targeted Therapy (2017) 2, e17017; doi:10.1038/sigtrans.2017.17; published online 7 July 2017

\section{INTRODUCTION}

The intestinal function declines with advancing age thereby causing an increasing incidence of gastrointestinal dysmotility in the geriatric population. ${ }^{1-5}$ Chronic constipation, especially the slow-transit constipation (STC), represents one of the most common gastrointestinal disorders in China and the western countries. Multifactorial aetiologies contribute to its initiation and development, including dysregulated enteric nervous system, ${ }^{6,7}$ altered gut microbiota composition, ${ }^{8,9}$ aberrant neurohormonal secretion, ${ }^{10-13}$ food pyramid and other patho-physiological abnormalities. ${ }^{14-17}$ Although anti-constipation strategies are continuously explored and updated, ${ }^{18}$ the morbidity of constipation irreversibly augments and this partly stems from an age-related rise in prevalence of constipation ${ }^{19-21}$ along with the accelerated global ageing. Using human specimens or animal models, many signalling pathways regulating gastrointestinal function were found altered during aging, such as neurokinin 2-mediated attenuation of tachykinin signalling ${ }^{15}$ and serotonergic signalling abnormalities. ${ }^{22}$ Besides, the morphology and number of villus and crypts, the secretion of anti-inflammatory cytokines and serotonin, the (re-)absorption of nutrients and fluids in colon are all altered compared to those of young. Although there is a lack of information regarding the precise mechanisms of ageing that leads to abnormalities in gastrointestinal motility, rejuvenating the ageing colon holds the promise to relief age-related colonic inertia.

Nicotinamide adenine dinucleotide (NAD) is one of the key coenzymes regulating many metabolic pathways, which is synthesized from extracellular precursors in the form of dinucleotides through two pathways, namely de novo pathway and salvage pathway. ${ }^{23,24}$ Recently, mounting evidence suggests that NAD biosynthesis declines with age $\mathrm{e}^{25-28}$ and therefore serving as a hallmark of senescence. ${ }^{29}$ In contrast, repletion of NAD to aged mice restores mitochondrial and stem cell function, but also rejuvenating tissue and extending lifespan. ${ }^{30-32}$ Moreover, in mammals $\mathrm{NAD}^{+}$also plays a pivotal role in modulating circadian clock $^{33}$ hepatosteatosis ${ }^{34}$ and other age-related metabolic complications. ${ }^{35}$ Although significant research has been carried out on various organs, colon remains to be the one of less studied organ during ageing and further the effect of ageing on colonic function is not fully understood. One notion that NAD functions as a purinergic, inhibitory neurotransmitter remains controversial. ${ }^{36-38}$ Nonetheless, there is a potential association between age-related alterations in intestinal motility and NAD level, however, it remains to be clarified whether declined NAD is a driver of the degeneration of normal colonic function, and whether repletion of NAD could rescue colonic degeneration of the aged.

To this end, we delineated the age-related alterations in the colon of young and old mice. In current STC models, we found a longer transit time and less faecal output in the aged mice partially due to reduced interstitial cells of Cajal (ICC) availability and augmented Aqp-mediated fluid reabsorption, although the Tph-5-HT-SR4 axis was more activated in aged colon compared to that of young controls. Intriguingly, age-related colon defect links to attenuated $\mathrm{NAD}^{+}$bioavailability. By pharmacological activation

${ }^{1}$ Institute of Ageing Research, Hangzhou Normal University School of Medicine, Hangzhou, China; ${ }^{2}$ Leibniz Institute for Age Research-Fritz Lipmann Institute (FLI), Jena, Germany and ${ }^{3}$ Department of Anorectal Surgery, the First Affiliated Hospital of Zhejiang University, Hangzhou, China.

Correspondence: Z Ju (zhenyuju@163.com) or Q Sheng (shengqinsong@163.com)

Received 1 December 2016; revised 24 February 2017; accepted 7 March 2017 
or inhibition of $\mathrm{NAD}^{+}$biosynthesis, we uncovered the protective role of $\mathrm{NAD}^{+}$in aged colon both in vitro and in vivo. Our results demonstrated that $\mathrm{NAD}^{+}$replenishment improved defecation in the aged mice and thus may represent a novel approach to protect against aging-related constipation.

\section{MATERIALS AND METHODS}

\section{Animal}

All procedures involving experimental animals were conducted in full accordance with approval by the Animal Care and Use Committee of Hangzhou Normal University. Young (3-month-old) and old (22 to 25month-old) C57Bl6J mice were maintained in a temperature-controlled room $\left(22 \pm 1{ }^{\circ} \mathrm{C}\right)$ on a 12 -h light/dark cycle with ad libitum access to food and water.

\section{Chemicals and antibodies}

Beta nicotinamide mononucleotide ( $\beta$-NMN) (BTO5) was purchased from BONTAC (Shenzhen, China). Nicotinamide phosphoribosyltransferase (Nampt) inhibitor (GMX1778) was from Selleck (Shanghai, China). Lomotil (Atropine-diphenoxylate) was purchased from Kangpu Pharmaceutical Ltd Co (Changzhou, China). Antibodies against serotonin transporter (ab181034), tryptophan hydroxylase (ab52954), Lgr5 (ab75850) and Nampt (ab45890) were purchased from Abcam (Cambridge, MA, USA). SR4 (sc376158), p15/16 (sc-377412) and goat anti-mouse IgG-FITC (sc-2010) were purchased from Santa Cruz Biotechnology (Dallas, TX, USA). APC antimouse CD117 (c-kit) (135108) was purchased from BioLegend (San Diego, CA, USA). Proliferating cell nuclear antigen (2586), GAPDH (5174) and Senescence $\beta$-Galactosidase Staining Kit (9860) were purchased from Cell Signaling Technology (Danvers, MA, USA). Goat anti-mouse IgG (31430), goat anti-rabbit IgG (31460) and TRIzol Reagent (15596-018) were purchased from Invitrogen (Carlsbad, CA, USA). EvaGreen Supermix (1725201 AP) was purchased from Bio-Rad Laboratories Inc (Hercules, CA, USA). PrimeScript first strand CDNA Synthesis Kit (6110A) was purchased from TaKaRa Bio Inc (Dalian, China). Nuclear Fast Red solution (N3020) and all other chemicals were purchased from Sigma-Aldrich (St Louis, MO, USA), unless otherwise stated.

\section{Establishment of slow-transit constipation animal model}

The atropine-diphenoxylate (Lomotil) was used to induce slow-transit constipation (STC) in mice according to previous reports ${ }^{39,40}$ with minor modification. Both young and old mice were assigned into two groups receiving either placebo (distilled water) or Lomotil at a dose of $5 \mathrm{mg} \mathrm{kg}^{-1}$ per day. STC group were gavaged with $100 \mu \mathrm{l}$ of distilled water+Lomotil $\left(5 \mathrm{mg} \mathrm{kg}^{-1}\right.$ ) once daily for 14 consecutive days. Vehicle group was treated with the same protocol except using equal amount of distilled water without Lomotil.

\section{Isolation and culture of primary colon epithelial cells}

Primary colon epithelial cells were isolated from freshly excised colon tissue following collagenase type A (Roche, Basel, Switzerland) digestion. Dulbecco's modified Eagle's medium (HyClone, Logan, UT, USA) containing $4.5 \mathrm{~g} \mathrm{I}^{-1}$ glucose, penicillin-streptomycin $\left(100 \mathrm{IU} \mathrm{ml}^{-1}\right.$ to $100 \mu \mathrm{g} \mathrm{ml}^{-1}$ ) and $10 \%$ foetal bovine serum (HyClone) was used for primary cell culture.

\section{Transit time}

Transit time was recorded as described ${ }^{41}$ with minor modification. Briefly, mice were fasted overnight and were orally gavaged with $200 \mu \mathrm{l}$ sterile solution of $6 \%$ carmine red (C110713, Aladdin) and $0.5 \%$ methylcellulose in water and placed in a new cage with no bedding. Refeeding started immediately post gavage, and all mice started eating within $5 \mathrm{~min}$. Since then mice were monitored every $5 \mathrm{~min}$ for production of a red faecal pellet. Transit time was recorded as the total number of minutes elapsed before production of the first red faecal pellet since mice started eating.

\section{Faecal output and water content}

Faecal output was analysed by separately housing each group of mice in a 24-h cycle for continuous 2 weeks. Typically three-five mice of each group were housed in one cage and average pellet output were calculated every $24 \mathrm{~h}$. Three cages of each group of mice were enrolled. Faecal pellets were removed at the end of every $24 \mathrm{~h}$ period and overall weight of wet pellet counts was obtained. The pellets were then left to dry at $50^{\circ} \mathrm{C}$ for $24 \mathrm{~h}$. Total wet and dry faecal weight was recorded. For the relative faecal water content, the differences between wet and dry faecal weight were divided by wet faecal weight, and this calculation from young mice was normalized as 1.0 .

\section{Colonic methylene blue staining}

The staining was performed as previously described. ${ }^{42}$ Briefly, small segments of colonic mucosa $(\sim 5 \times 1.0 \mathrm{~cm})$ were pinned out flat and fixed for $2 \mathrm{~h}$ in $2 \%$ paraformaldehyde in $0.1 \mathrm{M}$ sodium phosphate buffer $(\mathrm{pH} 7.4)$ at $4{ }^{\circ} \mathrm{C}$. The fixed tissue was stained for $3-5 \mathrm{~min}$ in $0.2 \%$ methylene blue in $0.1 \mathrm{M}$ sodium phosphate buffer $(\mathrm{pH} 7.4)$ and rinsed in fresh phosphate buffer at $4{ }^{\circ} \mathrm{C}$ for $30-60 \mathrm{~min}$ to allow more even distribution of the blue stain. The intact mucosa segments were placed luminal side up on microscope slides and observed with a low magnification ( $\times 4$ objective lens and $\mathrm{a} \times 10$ or $\times 15$ ocular lens).

\section{Senescence $\beta$-galactosidase staining}

The fresh isolated colons were fixed in fixation solution containing $2 \%$ formaldehyde and $0.2 \%$ glutaraldehyde in phosphate-buffered saline (PBS) for $1 \mathrm{~h}$, rinsed with PBS three times and then transferred to $30 \%$ sucrose and fixed for $24 \mathrm{~h}$. Then after, the tissues were embedded in Tissue-Tek O.C.T. Compound (Sakura Finetek, Torrance, CA, USA) for cutting to $6-\mu \mathrm{m}$ thick sections. Cryosections were fixed with $1 \times$ fixation buffer for 2 min, rinsed with PBS three times and incubated overnight at $37^{\circ} \mathrm{C}$ with the staining mixture supplied from the Senescence $\beta$-Galactosidase Staining Kit (Cell Signal, Danvers, MA, USA). The sections were counterstained with Nuclear Fast Red solution for nuclei labelling. Finally, the slides were scanned and photographed using a panoramic MIDI system from 3DHISTECH (Budapest, Hungary).

\section{Immunoblotting}

Total proteins were extracted from colon tissues using RIPA buffer (Applygen Technologies Inc, Beijing, China) supplemented with phosphatase and protease inhibitors (Roche). Equal amounts of proteins were separated by SDS-PAGE, and then transferred to polyvinylidene fluoride membranes (Millipore, Billerica, MA, USA). The membranes were reacted with primary antibody followed by secondary antibody. The immunoreactive bands were detected by Immun-Star WesternC chemiluminescence solutions (Bio-Rad Laboratories Inc).

\section{Immunohistochemistry and immunofluorescence staining}

For immunohistochemical staining, the fresh isolated colons were fixed in $4 \%$ paraformaldehyde solution for 24-48 h, embedded in paraffin, then cut to $6 \mu \mathrm{m}$ and mounted on glass slides. The slides were counterstained by haematoxylin $(4 \mathrm{~min})$, rinsed in running water $(5 \mathrm{~min})$, incubated with $1 \%$ eosin (aqueous, $1 \mathrm{~min}$ ) and rinsed with water until slide runs clear. Tissues were dehydrated sequentially with 70,95 and $100 \%$ ethanol, immersed in xylene and cover-slipped using a mounting medium, and images were captured using a panoramic MIDI system from 3DHISTECH.

For immunofluorescence staining, sections were deparaffinized, hydrated, washed, antigen unmasked and then blocked in 10\% normal goat serum for $1 \mathrm{~h}$, following with incubation overnight at $4{ }^{\circ} \mathrm{C}$ with SR4 or Nampt antibody. After washing three times in PBS with $1.5 \%$ normal blocking serum, the slides were incubated with goat anti-mouse IgG-FITC for $1 \mathrm{~h}$ at room temperature. The slides were then mounted by using Vectorshield Mounting Medium supplemented with $1.5 \mu \mathrm{g} \mathrm{ml}^{-1}$ diamidino-2-phenylindole ( $\mathrm{H}-1200$; Vector Laboratories, Burlingame, CA, USA). Immunofluorescent images were acquired using a senior upright Axio imager M2 fluorescence microscope from Carl Zeiss (Jena, Germany).

\section{Quantitative polymerase chain reaction}

Transcriptional expression levels of targeted genes in colon were assayed by quantitative polymerase chain reaction using primer sequences listed in Supplementary Table 1. Fresh excised colon tissues were snap frozen in liquid nitrogen and stored at $-80^{\circ} \mathrm{C}$. Total RNA was extracted using Trizol reagent (Life Technologies, Carlsbad, CA, USA) according to the manufacturer's instructions. Reverse transcription and quantitative polymerase chain reaction were performed using the PrimeScript first strand CDNA Synthesis Kit (TaKaRa Bio Inc) and EvaGreen Supermix 
(Bio-Rad Laboratories Inc) as per the manufacturers' instructions. The cycle threshold value determined for each RNA was normalized to the $\beta$-actin content to indicate relative RNA level.

\section{Serotonin measurement}

Whole colon was excised longitudinally and rinsed in cold PBS for five times to remove faeces. Longitudinal slim strip $(\sim 20 \mathrm{mg})$ of colon was cut for quantification. Colonic intracellular serotonin (5-HT) levels were measured using a commercial available ELISA kit (ADI-900-175, Enzo Life Sciences, Farmingdale, NY, USA) as per the manufacturers' instructions.

\section{Quantification of $\mathrm{NAD}^{+} / \mathrm{NADH}$ ratio}

Whole colon was excised longitudinally and rinsed in cold PBS for five times to remove faeces. Longitudinal slim strip $(\sim 20 \mathrm{mg})$ of colon was cut for quantification. Tissue $\mathrm{NAD}^{+} / \mathrm{NADH}$ ratio was analysed with a commercial NADH/NAD quantification kit (K337-100; Biovision, San Francisco, CA, USA) as per the manufacturers' instructions.

\section{Flow cytometry}

For flow cytometry analysis, APC c-Kit-labelled cell suspensions were analysed with an LSRFortessa cell analyser (BD Biosciences, Franklin Lakes, $\mathrm{NJ}$, USA). Rectangular regions were selected to define clusters with pink (presumed ICC).

\section{Statistics}

The data are represented as mean values \pm s.e.m. Student's $t$-tests or oneway ANOVA post Newman-Keuls multiple comparison tests were used where appropriate. $P<0.05$ was considered significant.

\section{RESULTS}

Defecation difficulty is accompanied with age-associated colonic degeneration

We compared defecation status of 3-month-old C57BL/6 (young) and 24-month-old (old) mice with identical breeding conditions. Mice body mass, food and drink intake were all similar between the two cohorts. To test whether the effect of (atropinediphenoxylate) Lomotil-induced slow-transit constipation (STC) on transit time was age-dependent, both young and old mice were assigned into two groups receiving either placebo (distilled water) or Lomotil at a dose of $5 \mathrm{mg} \mathrm{kg}^{-1}$ per day. After 2 weeks of gavage, no statistical difference of transit time between young and old mice was seen in vehicle group, but there was a significant elongated transit time in STC group versus that of vehicle group, as well as old mice treated with STC compared to that of young mice (Figure 1a). No sex-related differences were observed in current study (data not shown). The daily faecal output was significantly less in STC-treated groups in comparison to young groups, with an augmented reduction (old: $35.4 \%$ versus young: $29.6 \%$ ) in old group upon Lomotil treatment (Figure 1b). To evaluate whether these amplified defecation difficulty in STC old mice links to age-related colon degeneration, we next examined histological changes between young and old colons. Indeed, a significant reduction in villus number was found in the old colon versus the young colon (Figure 1c). Villus atrophy was seen in both vehicle and STC old mice (Figure 1d), while aged colon exhibited a higher frequency of hyperplasia independent of Lomotil treatment (Figure 1e). Lomotil-induced STC per se did not change the villus number or promote hyperplasia (data not shown). In addition, $\beta$-galactosidase (SA- $\beta$-gal) assay revealed more positive stains in aged colon while almost none in the young colon (Figure 1f), suggesting an increased incidence of age-associated colonic senescence. This was further confirmed by decreased intestinal stem cell marker Lgr5 and increased cell senescence marker cdkn2a (p16) seen in aged colon (Figures $1 \mathrm{~g}$ and h). Taken together, these data implicate that the defecation difficulty in the aged mice may stem from the degenerative colonic phenotypes.
Tph-5-HT-SR4 signalling axis is activated in aged colon

We next investigated the molecular mechanism linking defecation difficulty with age-associated colonic degeneration. Given serotonin signalling pathway plays a crucial role in regulating gut motility and defecation, ${ }^{9,12}$ we examined the serotonin release and downstream signalling in young and aged mice. Transcriptional expression of tryptophan hydroxylase 1 (Tph1), the predominant serotonin synthesis enzyme, together with Tph2, were upregulated, while serotonin reuptake transporter Slc6a4 (Sert) was downregulated in old colon in comparison to young colon (Figure 2a). Consistent with upregulated Tph expression, a higher serotonin level (Figure 2b) in parallel with enhanced type IV serotonin receptor (SR4) expression (Figure 2c) were seen in old colons. These results were further confirmed by immunoblotting analysis (Figure 2d), suggesting that the Tph-5-HT-SR4 signalling axis is activated in aged colon.

Decreased faecal water content and number of ICC in aged mice Given that increased 5-HT release led to enhanced aquaporin-3 (Aqp3) expression, ${ }^{43}$ which in turn promoted luminal dehydration, we speculated that increased 5 - $\mathrm{HT}$ in aged colon may lead to enhanced luminal dehydration via increasing local aquaporins expression. Indeed, expression levels of Aqp1, 3 and 11 were upregulated in aged colon while Aqp4 and 8 were unchanged (Figure $2 \mathrm{e}$ ), leading to a $\sim 17 \%$ faecal water content reduction compared to young patterns (Figure 2f). In identifying further correlation to defecation difficulty in aged mice, the intestinal pacemakers, ICC were considered as a possible target due to the ability to control enteric nervous system. ${ }^{44}$ Freshly prepared young and old colonic cells were stained with antibodies against ICC marker c-kit, and flow cytometry analysis displayed a significant reduction of c-kit positive population in aged colonocytes (Figure 2g; young: $2.65 \pm 0.17 \%$ versus old: $1.60 \pm 0.39 \%)$. Together, these data suggest that reduced viability of ICC while enhanced aquaporins (Aqp1, 3 and 11) may contribute to a less colonic motility and increased luminal dehydration in aged mice.

\section{NAD regulates colonic function and homoeostasis}

Since several lines of evidence demonstrate that oxidized form of $\mathrm{NAD}^{+}$or $\mathrm{NAD}^{+} / \mathrm{NADH}$ ratio is reduced in various age-related pathologies as well as during ageing process, ${ }^{45,46}$ we next assessed the major $\mathrm{NAD}^{+}$-generating enzyme expression in the salvage pathway to test whether a reduction in these enzymes accounts for the degeneration in the aged colon. Remarkably, transcription of nicotinamide mononucleotide adenylyltransferase 1, 2 and 3 (Nmnat1, 2 and 3), Nampt as well as nicotinamide riboside kinase 1 and 2 (Nmrk1 and 2) were all downregulated in old colon versus that in young colon (Figure 3a), which was further evidenced as aged colon exhibited a significantly lowered $\mathrm{NAD}^{+}$/ $\mathrm{NADH}$ ratio (Figure $3 \mathrm{~b}$ ). Interestingly, almost no positive immunofluorescent staining of Nampt was seen in those hyperplastic tissues from aged colon (Figure $3 c$ ), suggesting that lowered NAD level may correlate with vulnerability of hyperplasia/tumorigenesis. To further explore the correlation between $\mathrm{NAD}^{+}$level and colonic function, we administered the Nampt inhibitor GMX1778 to 2-month-old young mice intraperitoneally twice daily for 4 weeks. Injection of GMX1778 to young mice led to an ageing colon phenotype, including thinning of colonic muscle layer and villus atrophy (Figure $3 \mathrm{~d}$ ), with concurrent reductions in NAD content and faecal output (Figures $3 e$ and f). In contrast, 3-month continuous administration of $\beta-\mathrm{NMN}$, an $\mathrm{NAD}^{+}$precursor, restored colonic $\mathrm{NAD}^{+}$level to that of young mice (Figure $4 \mathrm{a}$ ) and improved colon function. Specifically, old mice receiving $\beta$-NMN favoured an increased colonic c-kit ${ }^{+}$population (Figure $4 \mathrm{~b}$ ) and significantly improved faecal output (Figure 4c). In addition, 
a

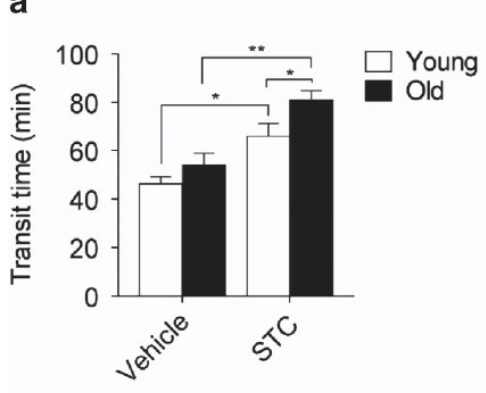

\section{b}

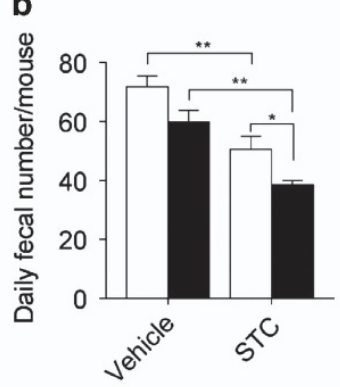

C

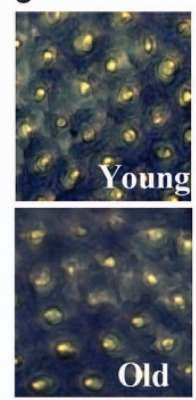

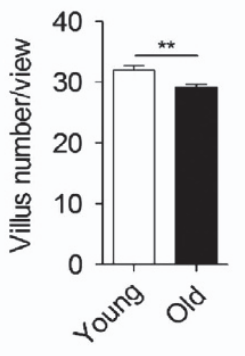

d
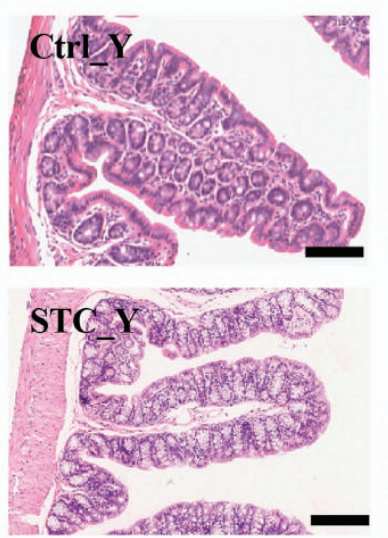

f

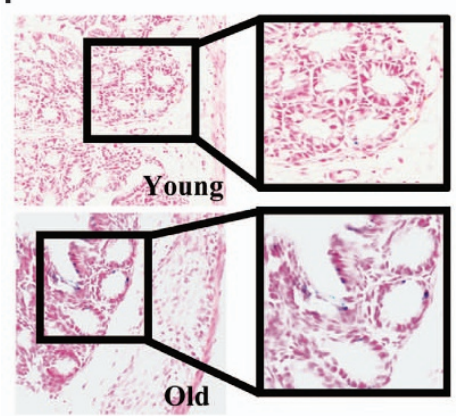

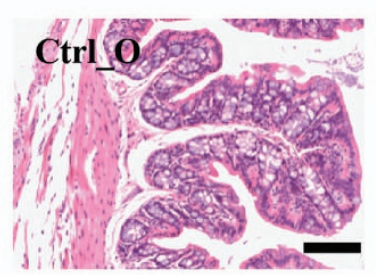

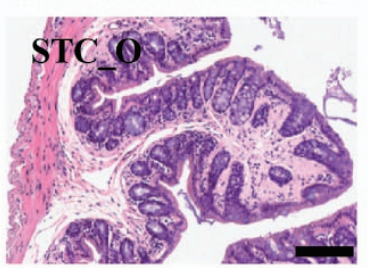

g

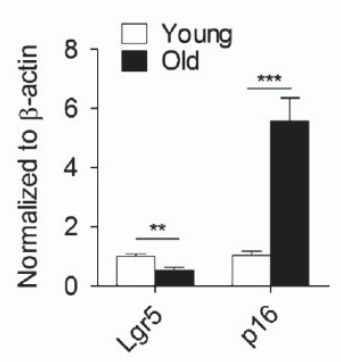

e
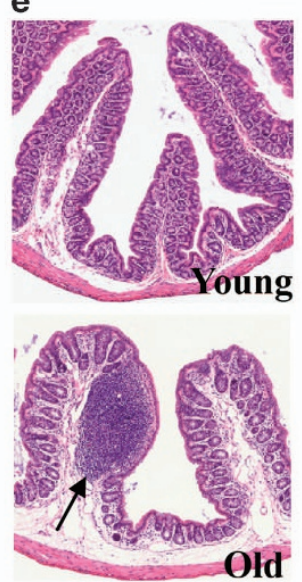

h
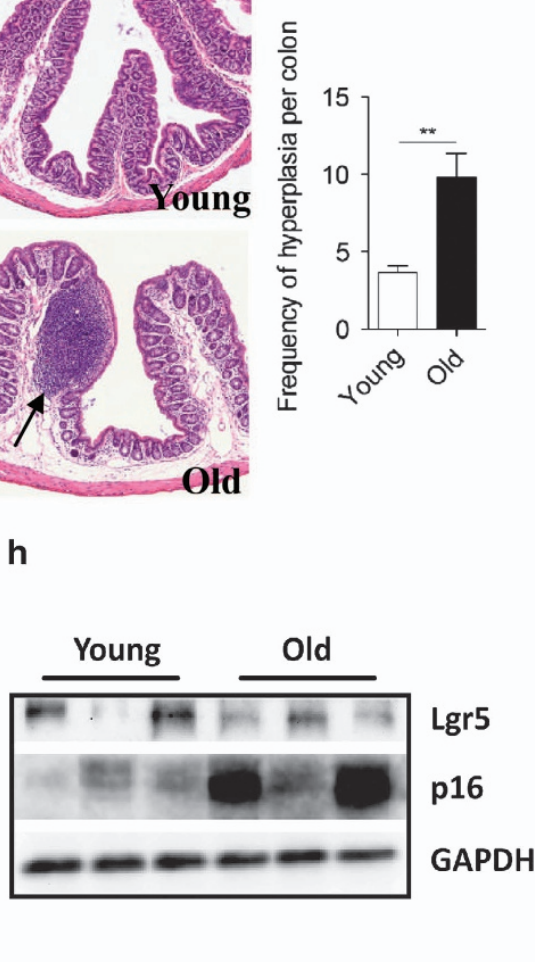

Figure 1. Defecation difficulty is accompanied with age-associated colonic degeneration. (a) Total transit time of young and old mice treated with or without Lomotil $(n=6)$. Data were presented as mean \pm s.e.m. ${ }^{*} P<0.05,{ }^{* *} P<0.01$. (b) Daily faecal number of young and old mice treated with or without Lomotil $(n=13-16)$. Data were presented as mean \pm s.e.m. ${ }^{*} P<0.05,{ }^{*} P<0.01$. (c) Representative images of methylene blue staining indicate decreased villus number in old colon. Villus numbers were counted in randomly selected six areas for each section. ${ }^{*} P<0.01$. (d) Representative images of haematoxylin and eosin staining from young and old colon treated with or without Lomotil. Bar $=100 \mu \mathrm{m}$. (e) Representative images of haematoxylin and eosin staining from young and old colon and calculation of hyperplasia ( $n=6$ ). Arrow denotes the occurrence site of hyperplasia. Data were presented as mean \pm s.e.m. ${ }^{* *} P<0.01$. (f) Representative images of senescenceassociated $\beta$-galactosidase (SA- $\beta$-gal) staining in cryosections from young and old colons. (g) Relative Lgr5 and cdkn2a (p16) mRNA expression in young and colons $(n=5-9)$. $\beta$-actin was used to normalize data. Data were presented as mean \pm s.e.m. ${ }^{* *} P<0.01,{ }^{* * *} P<0.001$. (h) Immunoblotting images of Lgr5 and p16 protein levels in young and old colons.

enhanced proliferation was seen in the isolated colon epithelial cells (Figure $4 \mathrm{~d}$ ) as well as proliferating cell nuclear antigenstained from aged mice receiving $\beta$-NMN (Figure 4e). Taken together, these data indicate that $\mathrm{NAD}^{+}$may serve as a regulator in colon homoeostasis during ageing and repletion of $\mathrm{NAD}^{+}$is able to improve colon function.

\section{DISCUSSION}

Colon ageing featured by altered morphology, dysregulated enteric nervous system, decreased secretion and propulsive motility, and other phenotypes, causes multiple gastrointestinal disorders in the elderly. In this study, we demonstrated that aged mice exhibited a spectrum of colon ageing phenotypes such as longer transit time and less faecal output partially due to reduced ICC availability and augmented aquaporin-mediated fluid reabsorption. Inhibiting NAD biosynthesis in young mice exhibited similar phenotype of aged colon. Intriguingly, these age-related colon defects could be partially rescued by $\mathrm{NAD}^{+}$repletion, thus, our results light up the veiled connection between $\mathrm{NAD}^{+}$and colon ageing.

Chronic constipation represents one of the most common problems among older people, exploration of its etiopathogenesis and emerging strategies of anti-constipation continuously 
a

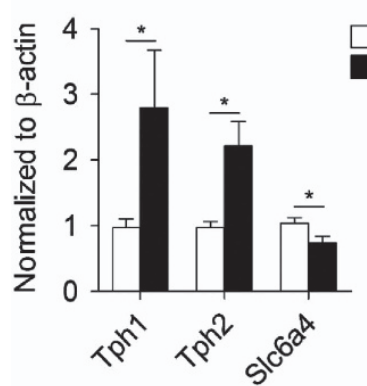

d

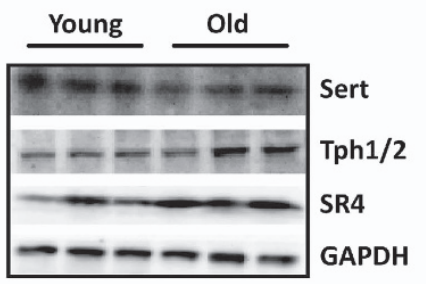

b

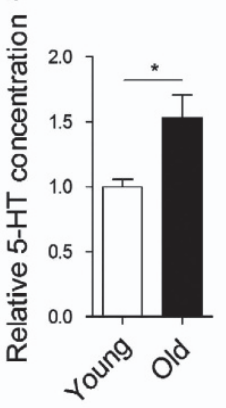

C

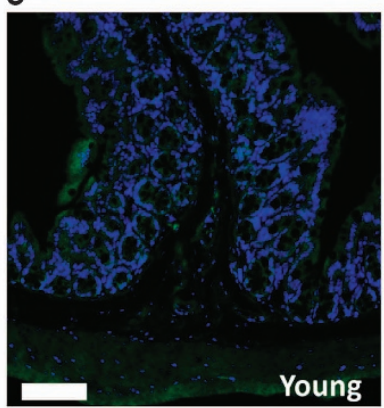

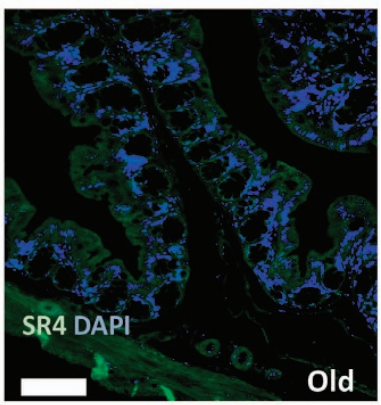

e

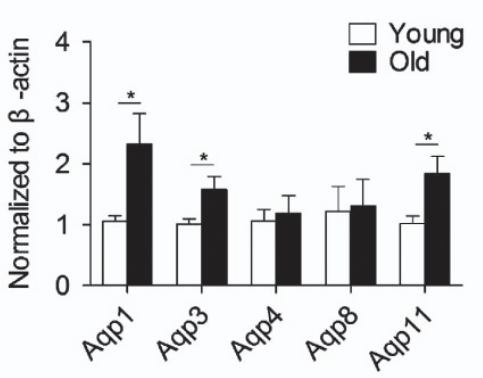

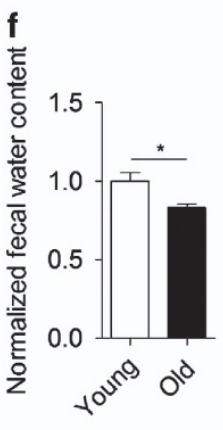

g

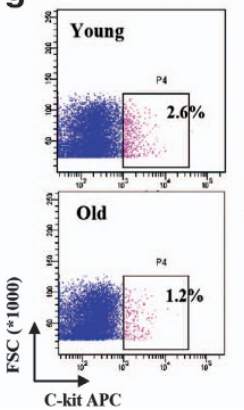

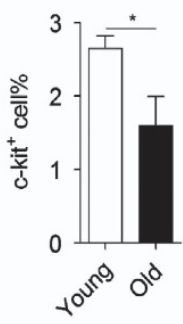

Figure 2. Decreased faecal water content and number of ICC contributes to defecation difficulty in aged mice. (a) Relative Tph1, Tph2 and Slc6a4 (sert) mRNA expression in young and colons $(n=8-12)$. $\beta$-actin was used to normalize data. Data were presented as mean \pm s.e.m. ${ }^{*} P<0.05$. (b) Relative serotonin (5-HT) release in young and colons $(n=4)$. ${ }^{*} P<0.05$. (c) Representative fluorescent images of SR4 expression in young and old colons. Bar $=100 \mu \mathrm{m}$. (d) Immunoblotting images of Sert, Tph1/2 and SR4 protein levels in young and old colons. (e) Relative aquaporins mRNA expression in young and colons $(n=4)$. $\beta$-actin was used to normalize data. Data were presented as mean $\pm s$.e.m. ${ }^{*} P<0.05$. (f) Relative faecal water content in young and old mice. Data were presented as mean \pm s.e.m. ${ }^{*} P<0.05$. (g) Representative flow cytometry plots and quantification of ICC (c-kit positive\% gated in pink in the rectangle) in young and old colons $(n=4)$. Data were presented as mean \pm s.e.m. ${ }^{*} P<0.05$.
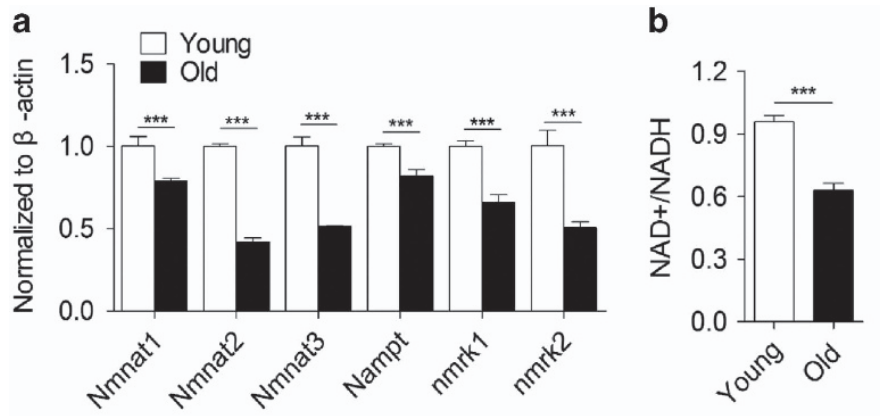

C

d
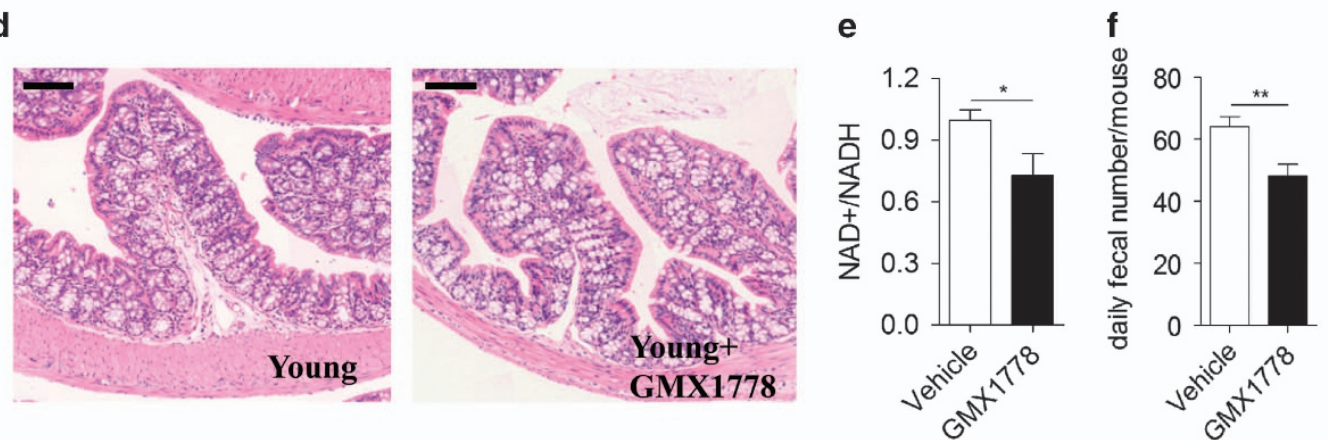

Figure 3. NAD regulates colonic function and homoeostasis. (a) Relative mRNA expression of salvage NAD biosynthetic enzymes in young and colons $(n=3)$. $\beta$-actin was used to normalize data. Data were presented as mean \pm s.e.m. ${ }^{* * *} p<0.001$. (b) Colonic NAD ${ }^{+} / \mathrm{NADH}^{*}$ ratio was analysed in young and colons $(n=4) .{ }^{* * *} P<0.001$. (c) Representative fluorescent images of Nampt expression in young and old colons. Bar $=100 \mu \mathrm{m}$. Irregularly dotted area in red denotes the occurrence site of hyperplasia. (d) Representative images of haematoxylin and eosin staining from young colons treated with or without specific Nampt inhibitor GMX1778. Bar $=100 \mu$ m. (e) Colonic NAD $/ \mathrm{NADH}^{\mathrm{ratio}}$ was analysed in young mice treated with or without Nampt inhibitor GMX1778 $(n=5)$. ${ }^{*} P<0.05$. (f) Daily faecal number of young mice treated with or without specific Nampt inhibitor GMX1778 $(n=6)$. ${ }^{* *} P<0.01$. 
a

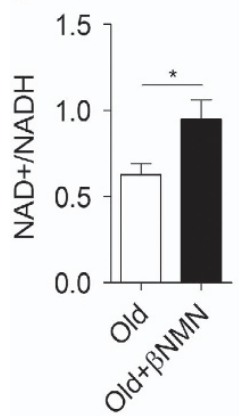

d

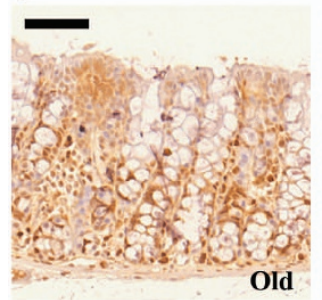

b

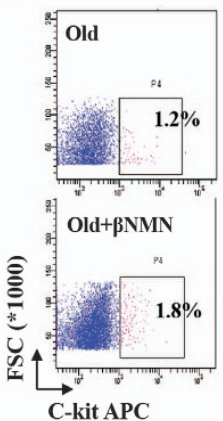

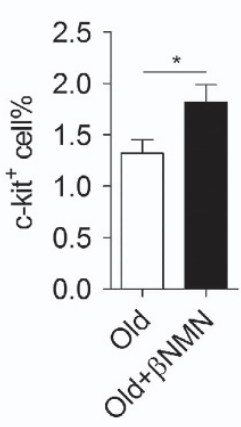

e

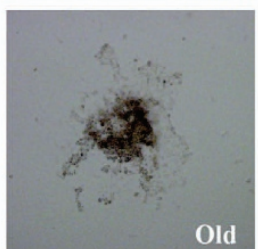

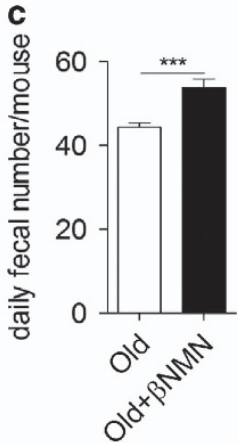

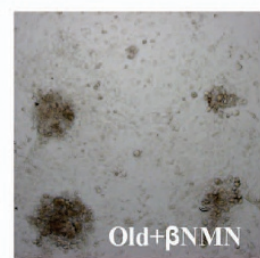

Figure 4. NAD repletion improves colonic function in aged mice. (a) Colonic NAD ${ }^{+}$NADH ratio was analysed in old mice treated with or without $\beta$-NMN for 3 months $(n=5)$. ${ }^{*} P<0.05$. (b) Representative flow cytometry plots and quantification of ICC (c-kit positive\% gated in pink in the rectangle) in old mice treated with or without $\beta$-NMN $(n=5)$. ${ }^{*} P<0.05$. (c) Daily faecal number of aged mice treated with or without $\beta$-NMN $(n=6)$. ${ }^{* * *} P<0.001$. (d) Representative images of proliferating cell nuclear antigen-stained colonic sections from aged mice treated with or without $\beta$-NMN. Bar $=50 \mu \mathrm{m}$. (e) Representative images of primary cultured colonic epithelial cells from aged mice treated with or without $\beta-\mathrm{NMN}$, on day 7 .

accelerate the relief of symptom, however, slow progress has been made due to the massive heterogeneity among humans and lack of suitable drugs that can fundamentally rejuvenate the ageing colon, plus the inevitable global ageing. Unlike human gastrointestinal studies puzzled by mixed genetic backgrounds, different geographic allocation and lifestyles, mice of high homogeneity fed standard chow and housed in an environment of constant temperature and humidity allow us to the explore the mechanisms underlying the age-related intestinal changes and its relevance to gastrointestinal disorders. By comparing young (3month-old) with old (24-month-old) mice, we observed significant colon degeneration in old mice, including villus atrophy, aberrant hyperplasia, increased senescence markers and defecation difficulty. Of note, the expression of Lgr5, the intestinal stem cell marker, was markedly reduced in old colon, which is thought to impair colon function by limiting the differentiation and proliferation of specific cell types regulating colon motility such as enterochromaffin cells, ${ }^{9}$ ICC, $^{44}$ epithelial cells ${ }^{47}$ and so on.

The Tph-5-HT-SR4 axis has been long studied in colon motility. ${ }^{48-50}$ Elevation of Tph expression and activity promotes the biosynthesis of 5-HT. Increased 5-HT concentration and binding to its receptor in colon further enhances bowel movement and accelerates colon transit. Abnormal Tph-5-HT-SR4 signalling has been found in diverticular disease, ${ }^{50}$ inflammatory bowel disease ${ }^{51}$ and irritable bowel syndrome. ${ }^{52}$ Previous observations have shown increased Tph1 activity, EC cell 5-HT content and $5-\mathrm{HT}$ release, but not decreased sert activity in patients with chronic constipation. ${ }^{11,53,54}$ In agreement, our results confirmed that aged colon exhibited an activated Tph-5-HT-SR4 signalling pathway with a decreased sert expression. Given activated Tph-5-HT-SR4 signalling leads to an enhanced intestinal propulsion, it seems paradoxical that aged colon exhibited a higher 5-HT level with a decreased stool output. One possible explanation is that $5-\mathrm{HT}$ receptor is desensitized upon long-term exposure of high 5-HT level, which leads to visceral hyposensitivity thereby a resulting reduction in stool frequency. Consistently, acutely increasing serotonergic activity with the selective serotonin reuptake inhibitor showed no effect on rectal motor function. ${ }^{55,56}$ In addition, serotonin was found to elevate aquaporin-3 expression in the colon using a morphine-induced constipation, we also found decreased faecal water content and shrinking number of ICC in aged mice, suggesting that altered 5HT signalling as a crucial driver to defecation problem in the aged.

Many studies have linked NAD with colorectal cancer, ${ }^{23}$ however, currently no direct evidence confirm the association between NAD level and defecation problem, although susceptibility of constipation increases with age concurrent with ageingrelated decreasing NAD level. ${ }^{15}$ Besides, a previous drug safety and efficacy study ${ }^{57}$ reported that NAD depleting drugs (mainly, Nampt inhibitors) caused various gastrointestinal symptoms, including constipation, suggesting NAD may play a role in regulating colon function. Indeed, our results demonstrated that administration of Nampt inhibitor GMX1778 was able to reduce faecal output and impair colon homoeostasis. In contrast, repletion of NAD via its precursor $\beta-N M N$ attenuated several colonic ageing phenotypes and improved defecation in old mice. One possible mechanism is that NAD serves as an enteric inhibitory neurotransmitter thereby contributing to neural regulation of colonic motility, ${ }^{37}$ although this remains controversial. ${ }^{38}$ Further study is of great significance to elaborate how NAD involves in the regulation of colonic motility. If so, it remains to be clarified if NAD plays a similar role in other colon dysfunction diseases like inflammatory bowel disease and irritable bowel syndrome.

In summary, our data indicate that ageing-related downregulation of colonic NAD level could be one of the causes of colon degeneration. Such lowered NAD biosynthesis in aged colon in parallel with increased tryptophan-5-HT signalling axis, decreased ICC cells and increased luminal dehydration could be one possible reason leading to the condition of constipation. By 
replenishing NAD, we observed a significant improvement of faecal output in the old mice, thus opening a novel avenue for the drug design to combat constipation.

\section{ACKNOWLEDGEMENTS}

This work was supported by grants from the Natural Science Foundation of Zhejiang province (Y13H030005) to QS, National Natural Science Foundation of China (81400221) and Hangzhou Normal University (PF14002004017) to XZ. The authors thank the assistance from Weiwei Yi for the flow cytometry analysis. $X Z, Z J$ and QS initiated the study and developed the concept of the paper; $X Z, Z J$ and QS designed the experiments; XZ, WS and YW performed experiments; XZ, ZJ and QS analysed and interpreted the data; and $\mathrm{XZ}, \mathrm{ZJ}$ and QS wrote the manuscript with help from $\mathrm{JA}$.

\section{COMPETING INTERESTS}

The authors declare no conflict of interest.

\section{REFERENCES}

1 Bannister JJ, Abouzekry L, Read NW. Effect of aging on anorectal function. Gut 1987; 28: 353-357.

2 Lovat LB. Age related changes in gut physiology and nutritional status. Gut 1996; 38: 306-309.

3 Madsen JL, Graff J. Effects of ageing on gastrointestinal motor function. Age Ageing 2004; 33: 154-159.

4 Fox JC, Fletcher JG, Zinsmeister AR, Seide B, Riederer SJ, Bharucha AE. Effect of aging on anorectal and pelvic floor functions in females. Dis Colon Rectum 2006; 49: $1726-1735$.

5 Rao SS, Go JT. Update on the management of constipation in the elderly: new treatment options. Clin Interv Aging 2010; 5: 163-171.

6 Burns AJ. Disorders of interstitial cells of Cajal. J Pediatr Gastroenterol Nutr 2007; 45(Suppl 2): S103-S106.

7 McClain JL, Grubisic V, Fried D, Gomez-Suarez RA, Leinninger GM, Sevigny J et al. $\mathrm{Ca} 2+$ responses in enteric glia are mediated by connexin- 43 hemichannels and modulate colonic transit in mice. Gastroenterology 2014; 146: 497-507 e1.

8 Ursell LK, Haiser HJ, Van Treuren W, Garg N, Reddivari L, Vanamala J et al. The intestinal metabolome: an intersection between microbiota and host. Gastroenterology 2014; 146: 1470-1476.

9 Yano JM, Yu K, Donaldson GP, Shastri GG, Ann P, Ma L et al. Indigenous bacteria from the gut microbiota regulate host serotonin biosynthesis. Cell 2015; 161: 264-276.

$10 \mathrm{Xu} \mathrm{L}$, Yu BP, Chen JG, Luo HS. Mechanisms mediating serotonin-induced contraction of colonic myocytes. Clin Exp Pharmacol Physiol 2007; 34: 120-128.

11 Costedio MM, Coates MD, Brooks EM, Glass LM, Ganguly EK, Blaszyk H et al. Mucosal serotonin signaling is altered in chronic constipation but not in opiateinduced constipation. Am J Gastroenterol 2010; 105: 1173-1180.

12 Mawe GM, Hoffman JM. Serotonin signalling in the gut--functions, dysfunctions and therapeutic targets. Nat Rev Gastroenterol Hepatol 2013; 10: 473-486.

13 D'Addio F, La Rosa S, Maestroni A, Jung P, Orsenigo E, Ben Nasr M et al. Circulating IGF-I and IGFBP3 levels control human colonic stem cell function and are disrupted in diabetic enteropathy. Cell Stem Cell 2015; 17: 486-498.

14 Tong WD, Liu BH, Zhang LY, Xiong RP, Liu P, Zhang SB. Expression of c-kit messenger ribonucleic acid and c-kit protein in sigmoid colon of patients with slow transit constipation. Int J Colorectal Dis 2005; 20: 363-367.

15 Patel BA, Patel N, Fidalgo S, Wang C, Ranson RN, Saffrey MJ et al. Impaired colonic motility and reduction in tachykinin signalling in the aged mouse. Exp Gerontol 2014; 53: 24-30.

16 El-Salhy M, Hatlebakk JG, Hausken T. Reduction in duodenal endocrine cells in irritable bowel syndrome is associated with stem cell abnormalities. World J Gastroenterol 2015; 21: 9577-9587.

17 Zhu F, Xu S, Zhang Y, Chen F, Ji J, Xie G. Total glucosides of paeony promote intestinal motility in slow transit constipation rats through amelioration of interstitial cells of Cajal. PloS ONE 2016; 11: e0160398.

18 Rao SS, Rattanakovit K, Patcharatrakul T. Diagnosis and management of chronic constipation in adults. Nat Rev Gastroenterol Hepatol 2016; 13: 295-305.

19 Hall KE, Proctor DD, Fisher L, Rose S. American gastroenterological association future trends committee report: effects of aging of the population on gastroenterology practice, education, and research. Gastroenterology 2005; 129: 1305-1338.

20 Bitar K, Greenwood-Van Meerveld B, Saad R, Wiley JW. Aging and gastrointestinal neuromuscular function: insights from within and outside the gut. Neurogastroenterol Motil 2011; 23: 490-501.
21 Tran L, Greenwood-Van Meerveld B. In a non-human primate model, aging disrupts the neural control of intestinal smooth muscle contractility in a regionspecific manner. Neurogastroenterol Motil 2014; 26: 410-418.

22 Gershon MD, Tack J. The serotonin signaling system: from basic understanding to drug development for functional Gl disorders. Gastroenterology 2007; 132: 397-414.

23 Chiarugi A, Dolle C, Felici R, Ziegler M. The NAD metabolome--a key determinant of cancer cell biology. Nat Rev Cancer 2012; 12: 741-752.

24 Nikiforov A, Kulikova V, Ziegler M. The human NAD metabolome: functions, metabolism and compartmentalization. Crit Rev Biochem Mol Biol 2015; 50 284-297.

25 Braidy N, Guillemin GJ, Mansour H, Chan-Ling T, Poljak A, Grant R. Age related changes in NAD+ metabolism oxidative stress and Sirt1 activity in wistar rats. PloS One 2011; 6: e19194.

26 Massudi H, Grant R, Braidy N, Guest J, Farnsworth B, Guillemin GJ. Age-associated changes in oxidative stress and NAD+ metabolism in human tissue. PloS One 2012; 7: e42357.

27 Verdin $\mathrm{E} . \mathrm{NAD}(+)$ in aging, metabolism, and neurodegeneration. Science 2015 350: $1208-1213$.

28 Camacho-Pereira J, Tarrago MG, Chini CC, Nin V, Escande C, Warner GM et al CD38 dictates age-related NAD decline and mitochondrial dysfunction through an SIRT3-dependent mechanism. Cell Metab 2016; 23: 1127-1139.

29 Lopez-Otin C, Blasco MA, Partridge L, Serrano M, Kroemer G. The hallmarks of aging. Cell 2013; 153: 1194-1217.

30 Belenky P, Racette FG, Bogan KL, McClure JM, Smith JS, Brenner C. Nicotinamide riboside promotes Sir2 silencing and extends lifespan via Nrk and Urh1/Pnp1/ Meu1 pathways to NAD+. Cell 2007; 129: 473-484.

31 Mouchiroud L, Houtkooper RH, Moullan N, Katsyuba E, Ryu D, Canto C et al. The $\mathrm{NAD}(+)$ /sirtuin pathway modulates longevity through activation of mitochondrial UPR and FOXO signaling. Cell 2013; 154: 430-441.

32 Imai S, Guarente L. NAD+ and sirtuins in aging and disease. Trends Cell Biol 2014 24: 464-471.

33 Ramsey KM, Yoshino J, Brace CS, Abrassart D, Kobayashi Y, Marcheva B et al. Circadian clock feedback cycle through NAMPT-mediated NAD+ biosynthesis. Science 2009; 324: 651-654.

34 Gariani K, Menzies KJ, Ryu D, Wegner CJ, Wang X, Ropelle ER et al. Eliciting the mitochondrial unfolded protein response by nicotinamide adenine dinucleotide repletion reverses fatty liver disease in mice. Hepatology 2016; 63: 1190-1204

35 Yoshino J, Mills KF, Yoon MJ, Imai S. Nicotinamide mononucleotide, a key NAD(+) intermediate, treats the pathophysiology of diet- and age-induced diabetes in mice. Cell Metab 2011; 14: 528-536.

36 Mutafova-Yambolieva VN, Hwang SJ, Hao X, Chen H, Zhu MX, Wood JD et al. Betanicotinamide adenine dinucleotide is an inhibitory neurotransmitter in visceral smooth muscle. Proc Natl Acad Sci USA 2007; 104: 16359-16364.

37 Hwang SJ, Durnin L, Dwyer L, Rhee PL, Ward SM, Koh SD et al. beta-nicotinamide adenine dinucleotide is an enteric inhibitory neurotransmitter in human and nonhuman primate colons. Gastroenterology 2011; 140: 608-17 e6.

38 Goyal RK. Evidence for beta-nicotinamide adenine dinucleotide as a purinergic inhibitory neurotransmitter in doubt. Gastroenterology 2011; 141: e27.

39 Xu HM, Wei W, Jia XY, Chang Y, Zhang L. Effects and mechanisms of total glucosides of paeony on adjuvant arthritis in rats. J Ethnopharmacol 2007; 109: $442-448$.

40 Xu J, Zhou X, Chen C, Deng Q, Huang Q, Yang J et al. Laxative effects of partially defatted flaxseed meal on normal and experimental constipated mice. BMC Complement Altern Med 2012; 12: 14.

41 Li Z, Chalazonitis A, Huang YY, Mann JJ, Margolis KG, Yang QM et al. Essential roles of enteric neuronal serotonin in gastrointestinal motility and the development/ survival of enteric dopaminergic neurons. J Neurosci 2011; 31: 8998-9009.

42 Pretlow TP, Barrow BJ, Ashton WS, O'Riordan MA, Pretlow TG, Jurcisek JA et al. Aberrant crypts: putative preneoplastic foci in human colonic mucosa. Cancer Res 1991; 51: 1564-1567.

43 Kon R, Ikarashi N, Hayakawa A, Haga Y, Fueki A, Kusunoki $Y$ et al. Morphineinduced constipation develops with increased aquaporin-3 expression in the colon via increased serotonin secretion. Toxicol Sci 2015; 145: 337-347.

44 Wedel T, Spiegler J, Soellner S, Roblick UJ, Schiedeck TH, Bruch HP et al. Enteric nerves and interstitial cells of Cajal are altered in patients with slow-transit constipation and megacolon. Gastroenterology 2002; 123: 1459-1467.

45 Srivastava S. Emerging therapeutic roles for NAD(+) metabolism in mitochondria and age-related disorders. Clin Transl Med 2016; 5: 25.

46 Frederick DW, Loro E, Liu L, Davila A Jr., Chellappa K, Silverman IM et al. Loss of NAD homeostasis leads to progressive and reversible degeneration of skeletal muscle. Cell Metab 2016; 24: 269-282. 
47 Guarino M, Cheng L, Cicala M, Ripetti V, Biancani P, Behar J. Progesterone receptors and serotonin levels in colon epithelial cells from females with slow transit constipation. Neurogastroenterol Motil 2011; 23: 575-e210.

48 Oosterbosch L, von der Ohe M, Valdovinos MA, Kost L, Phillips SF, Camilleri M. Effects of serotonin on rat ileocolonic transit and fluid transfer in vivo: possible mechanisms of action. Gut 1993; 34: 794-798.

49 Hoffman JM, Tyler K, MacEachern SJ, Balemba OB, Johnson AC, Brooks EM et al. Activation of colonic mucosal 5-HT(4) receptors accelerates propulsive motility and inhibits visceral hypersensitivity. Gastroenterology 2012; 142: 844-54 e4.

50 Bottner M, Barrenschee M, Hellwig I, Harde J, Egberts JH, Becker T et al. The enteric serotonergic system is altered in patients with diverticular disease. Gut 2013; 62: 1753-1762.

51 Linden DR, Foley KF, McQuoid C, Simpson J, Sharkey KA, Mawe GM. Serotonin transporter function and expression are reduced in mice with TNBS-induced colitis. Neurogastroenterol Motil 2005; 17: 565-574.

52 Coates MD, Mahoney CR, Linden DR, Sampson JE, Chen J, Blaszyk $\mathrm{H}$ et al. Molecular defects in mucosal serotonin content and decreased serotonin reuptake transporter in ulcerative colitis and irritable bowel syndrome. Gastroenterology 2004; 126: 1657-1664.

53 Lincoln J, Crowe R, Kamm MA, Burnstock G, Lennard-Jones JE. Serotonin and 5-hydroxyindoleacetic acid are increased in the sigmoid colon in severe idiopathic constipation. Gastroenterology 1990; 98(5 Pt 1): 1219-1225.

54 Miwa J, Echizen H, Matsueda K, Umeda N. Patients with constipationpredominant irritable bowel syndrome (IBS) may have elevated serotonin concentrations in colonic mucosa as compared with diarrhea-predominant patients and subjects with normal bowel habits. Digestion 2001; 63: 188-194.

55 Kilkens TO, Honig A, Fekkes D, Brummer RJ. The effects of an acute serotonergic challenge on brain-gut responses in irritable bowel syndrome patients and controls. Aliment Pharmacol Ther 2005; 22: 865-874.

56 van Nieuwenhoven MA, Kilkens TO. The effect of acute serotonergic modulation on rectal motor function in diarrhea-predominant irritable bowel syndrome and healthy controls. Eur J Gastroenterol Hepatol 2012; 24: 1259-1265.

57 von Heideman A, Berglund A, Larsson R, Nygren P. Safety and efficacy of NAD depleting cancer drugs: results of a phase I clinical trial of CHS 828 and overview of published data. Cancer Chemother Pharmacol 2010; 65: 1165-1172.

This work is licensed under a Creative Commons Attribution 4.0 International License. The images or other third party material in this article are included in the article's Creative Commons license, unless indicated otherwise in the credit line; if the material is not included under the Creative Commons license, users will need to obtain permission from the license holder to reproduce the material. To view a copy of this license, visit http://creativecommons.org/licenses/ by/4.0/

(c) The Author(s) 2017

Supplementary Information accompanies the paper on the Signal Transduction and Targeted Therapy website (http://www.nature.com/sigtrans) 